\title{
Evaluation of neutron activation cross section data for energies up to $150 \mathrm{MeV}$
}

\author{
P. Pereslavtsev ${ }^{1, \mathrm{a}}$, U. Fischer ${ }^{1}$, and S. Sudar ${ }^{2}$ \\ 1 Forschungszentrum Karlsruhe, Institut für Reaktorsicherheit, Postfach 3640, 76021 Karlsruhe, Germany \\ 2 Institute of Experimental Physics, University of Debrecen, 4010 Debrecen Pf. 105, Hungary
}

\begin{abstract}
A new version of the activation data library for incident neutron energies to $150 \mathrm{MeV}$ is under elaboration. The evaluated reaction cross sections are based on GNASH nuclear model calculations. Two new global optical model potentials were elaborated for incident helions and tritons and incident energies up to $220 \mathrm{MeV}$. For the new library a part of low energy data were accepted from EAF-2005 neutron activation data library. The evaluated data are stored in ENDF-6 formatted files suitable for further NJOY processing.
\end{abstract}

\section{Introduction}

A completely new version of the neutron activation data library IEAF-2001 [1] is currently developed in a collaboration between Forschungszentrum Karlsuhe, Germany, University of Debrecen, Hungary and the Obninsk State Technical University, Russia. This library will contain neutron induced activation and transmutation cross sections for nuclides with $\mathrm{Z}=1-84$. The neutron energy range will extend from thermal energy up to $1 \mathrm{GeV}$. Above $150 \mathrm{MeV}$ the data are based on intranuclear cascade model calculations. Below $150 \mathrm{MeV}$, the cross sections are evaluated on the basis of nuclear model calculations. This work is devoted to the evaluation of the cross section data from thermal energies to $150 \mathrm{MeV}$.

\section{Nuclear models and tools used}

\section{Evaluation procedure}

The flowchart of the evaluation procedure is shown in figure 1. The core routine for cross section calculations is GNASH code [2] which is used for calculations from 0.001 to $150 \mathrm{MeV}$ incident neutron energies. Preparation of the new activation data library assumes numerous GNASH calculations for nuclei with atomic numbers up to 84 . To prepare detailed input files for GNASH we make use of RIPL-2 data [3]. Transmission coefficients along with total, elastic scattering and reaction cross sections are calculated with ECIS96 [4] code that is very powerful for optical model calculations. Additional input file is prepared to account for collective excitations in $\left(\mathrm{n}, \mathrm{n}^{\prime}\right)$ channel. The cross sections for collective states with various multi-polarities are calculated with ECIS code as well.

For nuclear cross sections evaluation we considered the following data: GNASH results; EAF-2005 activation data library [5]; experimental data from EXFOR library and literature; systematics predictions at $\sim 14.5 \mathrm{MeV}$.

Analysis, based on the comparison of these data, allows to make the best fit of the excitation functions. The excitation

${ }^{a}$ Presenting author, e-mail: pavel.pereslavtsev@irs.fzk.de

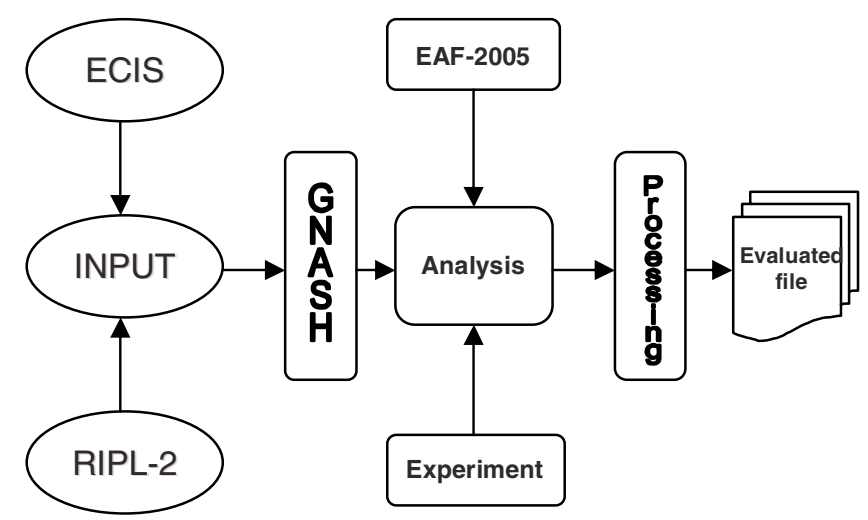

Fig. 1. Flowchart of the activation data evaluation procedure.

functions for numerous reaction channels are then processed in final ENDF-6 formatted activation data file. The final data processing assumes a high degree of automation because the number of data files (library content) reaches 684 .

\section{Optical model calculations with ECIS96}

The choice of the optical model potential (OMP) for activation calculations is crucial. We considered decay of a nucleus with emission of neutrons, protons, deuterons, tritons, helions $\left({ }^{3} \mathrm{He}\right)$ and alphas. The following OMPs were used: for $n$ and $p-$ by Koning \& Delaroche [6]; for d - by Nankai university, China [7]; for t, ${ }^{3} \mathrm{He}-$ newly elaborated OMP [8]; for alpha $s$ - by Avrigeanu \& Hodgson [9].

We elaborated an automated procedure around ECIS96 for the preparation of the transmission coefficients for GNASH calculations using ECIS96 as a subroutine. Transmission coefficients as well as total, elastic scattering and reaction cross sections are calculated for the arbitrary energy mesh for all particles.

For the description of the collective excitations we considered the excitation of the giant resonances with four different multi-polarities as proposed by Kalbach [10]. The cross section for each resonance are calculated with ECIS96 for all 
Table 1. OMP parameters for ${ }^{3} \mathrm{He}$ and tritons.

\begin{tabular}{cc}
\hline${ }^{3} \mathrm{He}$ & tritons \\
\hline $\mathrm{V}_{0}=138.1-35.54 \xi$ & $\mathrm{V}_{0}=138.1-35.54 \xi$ \\
$\alpha_{V}=-(0.00258-0.0004 \xi)$ & $\alpha_{V}=-(0.00258-0.0004 \xi)$ \\
$\mathrm{r}_{V}=1.1269+0.4804 \xi$ & $\mathrm{r}_{V}=1.0272+0.4804 \xi$ \\
$\mathrm{a}_{V}=0.8559-0.333 \xi$ & $\mathrm{a}_{V}=0.8559-0.238 \xi$ \\
\hline $\mathrm{W}_{0}=24.138+5.127 \xi$ & $\mathrm{W}_{0}=24.541+5.127 \xi$ \\
$\varepsilon_{W}=0.0978+0.4252 \xi$ & $\varepsilon_{W}=0.0978+0.4252 \xi$ \\
$\alpha_{W}=-(0.002018-0.001587 \xi)$ & $\alpha_{W}=-(0.002018-0.001587 \xi)$ \\
$\mathrm{r}_{W}=1.6292+0.93 \xi$ & $\mathrm{r}_{W}=1.6292+0.93 \xi$ \\
$\mathrm{a}_{W}=0.749-0.3385 \xi$ & $\mathrm{a}_{W}=0.749-0.3385 \xi$ \\
\hline
\end{tabular}

neutron incidence energies and then it is broadened in energy assuming a Gaussian distribution.

\section{OMPs for helions and tritons}

There is the no global OMP for helions and tritons suitable for a wide energy range from several to $150 \mathrm{MeV}$ and covering atomic numbers from very small to 84 . Well known OMPs by Becchetti \& Greenlees [11] can be used only for energies up to $40 \mathrm{MeV}$. A possible solution here is a folding method [12], where an OMP for complex particles is built on the basis of existing OMPs for neutrons and protons. In spite of the flexibility of the folding method applications (depends only on availability of neutron and proton OMPs), its predictive power is not sufficient [13].

The experimental data base for ${ }^{3} \mathrm{He}$ covers energies from several to hundreds $\mathrm{MeV}$. New measurements for ${ }^{3} \mathrm{He}$ reaction cross sections and energies from 96 to $168 \mathrm{MeV}$ were published in [14]. In this work the relativistic OMPs for ${ }^{12} \mathrm{C},{ }^{40} \mathrm{Ca}$, ${ }^{58} \mathrm{Ni}$ and ${ }^{208} \mathrm{~Pb}$ were elaborated, the incident energies being from tens to $217 \mathrm{MeV}$. The following Woods-Saxon form of the OMP was proposed:

$$
\begin{gathered}
V(E)=V_{0}\left(1+\alpha_{v} E_{c . m .}\right), \\
W(E)=W_{0}\left[1-\exp \left(-\varepsilon_{W} E_{c . m .}\right)\right]\left(1+\alpha_{W} E_{c . m .}\right),
\end{gathered}
$$

where $E_{c . m}$ is the kinetic energy of a particle in a center-ofmass system.

We fitted OMP parameters derived in [14] to get a global OMP for ${ }^{3} \mathrm{He}$. For tritons we considered the same form of the OMP. Presented in table 1 are the fitted parameters of the new global OMPs for hellions and tritons. Here $\xi$ is $(N Z) / A$ and the reduced radius for Coulomb potential is $r_{C}=1.3 \mathrm{fm}$. Particle energy in equations (1) and (2) is given in $\mathrm{MeV}$ and geometry parameters $r_{V, W}$ and $a_{V, W}$ are in fm.

The new global OMPs (eqs. (1), (2) and table 1) are assumed to be valid for incident energies of hellions and tritons $E_{c . m}<200 \mathrm{MeV}$ and atomic numbers from 4 to 84 . Results of the optical model calculations with ECIS96 code for hellions and tritons are shown in figures 2-4. For comparison we present results obtained with OMPs from $[13,16]$ and the folding approach. Reaction cross section for incident ${ }^{3} \mathrm{He}$ calculated with folding method for construction of the OMP is well underestimated.

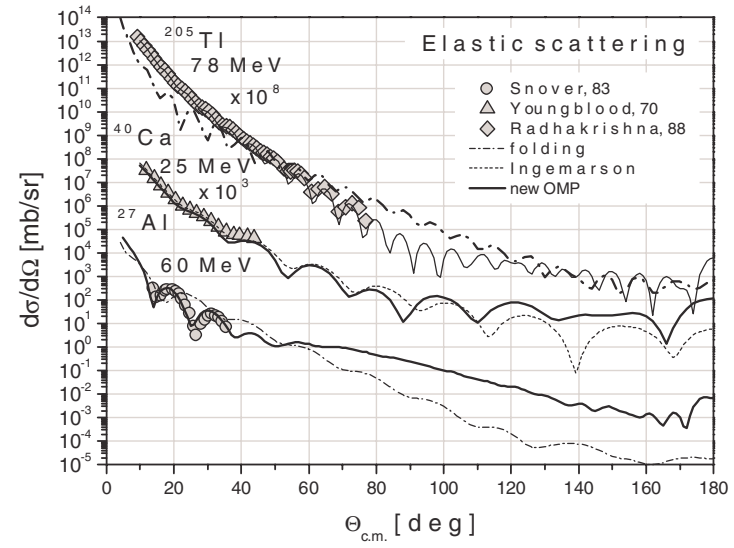

Fig. 2. Elastic scattering of ${ }^{3} \mathrm{He}$ by ${ }^{27} \mathrm{Al},{ }^{40} \mathrm{Ca},{ }^{205} \mathrm{Tl}$.

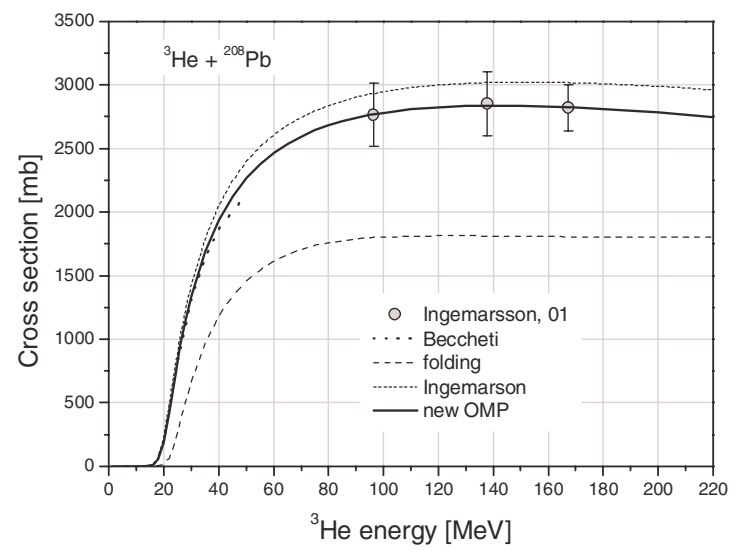

Fig. 3. Reaction cross section for ${ }^{3} \mathrm{He}+{ }^{208} \mathrm{~Pb}$.

\section{Nuclear model calculations with GNASH}

GNASH utilizes the Hauser-Feshbach model for multiple particle emissions through statistical processes, the exciton model of Kalbach [15] for single particle and the model of Chadwick [16] for multiple particle pre-equilibrium reactions. For compound nuclear reactions we used the Ignatyuk form of the Fermi-gas model with energy-dependent level density parameters [17]. Gamma-ray transmission coefficients were calculated using the Kopecky and Uhl model [18]. With GNASH we calculated total nuclide production cross sections rather than cross sections for the definite nucleus decay. In addition isomer formation and gas production cross sections were calculated.

Since the evaluation covers incident neutron energies up to $150 \mathrm{MeV}$ we accounted for in the calculations as many as possible reaction paths. The number of residual nuclides considered was limited by a maximum atomic number difference of $\Delta \mathrm{Z}=8$ and a maximum atomic mass difference of $\Delta \mathrm{A}=20$ with respect to the target nucleus.

\section{Activation data library}

\section{Data evaluation}

The new library is based mainly on GNASH results. Nevertheless we try to take advantage from the usage of the 


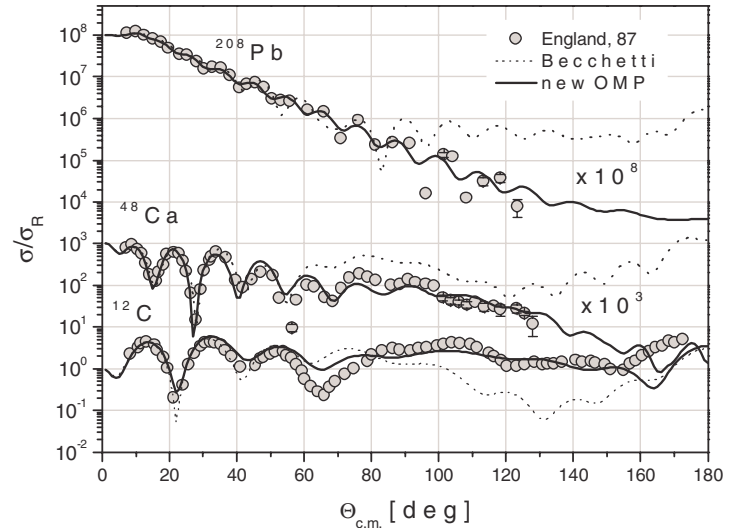

Fig. 4. Elastic scattering of $33 \mathrm{MeV}$ tritons by ${ }^{12} \mathrm{C},{ }^{48} \mathrm{Ca},{ }^{208} \mathrm{~Pb}$.

already available evaluations for some reaction channels. EAF-2005 activation data library is the most advanced one to be considered for inclusion in the newly developed library. The EAF-2005 library evaluated data demonstrate high quality especially below $20 \mathrm{MeV}$. For those reaction channels that we accept for the new library the adjustment with GNASH results is made usually at $20 \mathrm{MeV}$ or in some cases (where EAF-2005 are very reliable) at $60 \mathrm{MeV}$.

Presented in figures 5-9 are examples of the evaluated data for various target nuclides and reaction channels. We cut some excitation functions in figures only for convenience for evaluated data representation. In spite of the high quality of the EAF-2005 data we perform comparison of the GNASH and EAF-2005 results with experimental data if any. We performed new evaluation, for example, for ${ }^{150} \mathrm{Sm}(\mathrm{n}, 2 \mathrm{n}){ }^{149} \mathrm{Sm}$ cross section (fig. 5) fitting the latest experimental data. The new evaluation for this reaction seams to be more reasonable compared to EAF-2005 results. An example of the isomer formation cross section evaluation is given in figure 6 for ${ }^{153} \mathrm{Eu}(\mathrm{n}, 2 \mathrm{n}){ }^{152 \mathrm{~m} 1} \mathrm{Eu}$ reaction. For this reaction we performed normalization of the GNASH results to the latest experimental data. The use of the new OMPs for tritons and helions enables more accurate GNASH calculations compared to the folding approach. In figure 7 we present new evaluation for ${ }^{115} \operatorname{In}(\mathrm{n}, \mathrm{x}){ }^{113} \mathrm{Cd}$ cross section. The $(\mathrm{n}, \mathrm{t})$ reaction is dominant here below $20 \mathrm{MeV}$ neutron incident energy. The GNASH results were used for the ${ }^{115} \operatorname{In}(\mathrm{n}, \mathrm{x}){ }^{113} \mathrm{Cd}$ cross section evaluation without modifications. The evaluated high energy cross sections for ${ }^{209} \mathrm{Bi}(\mathrm{n}, \mathrm{xn})$ are shown in figure 8 .

The new data library contains as an important part gas production cross sections for $\mathrm{H},{ }^{2} \mathrm{H},{ }^{3} \mathrm{H},{ }^{3} \mathrm{He}, \mathrm{He}$. In figure 9 we give the comparison between different evaluations for $\mathrm{Fe}(\mathrm{n}, \mathrm{x} \alpha)$ cross section. No evaluation fully reproduces measured high energy data for $\mathrm{Fe}(\mathrm{n}, \mathrm{x} \alpha)$.

\section{Pointwise data format}

The format for data representation is ENDF-6. A very short description is given in MF $=1 \mathrm{MT}=451$. Standard ENDF-6 switches are applied to identify target nucleus, for example, instable nuclide, isomeric state, etc. The section $\mathrm{MF}=2$ MT = 151 (resonance parameters) is absolutely necessary for

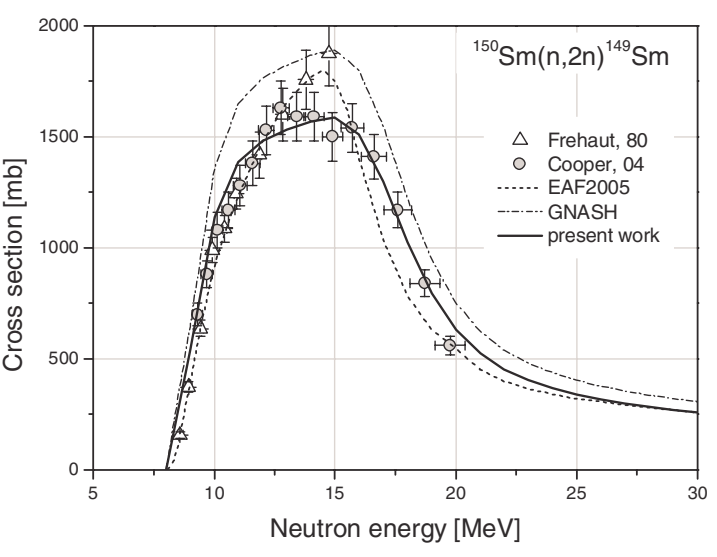

Fig. 5. ${ }^{150} \mathrm{Sm}(\mathrm{n}, 2 \mathrm{n}){ }^{149} \mathrm{Sm}$ cross section.

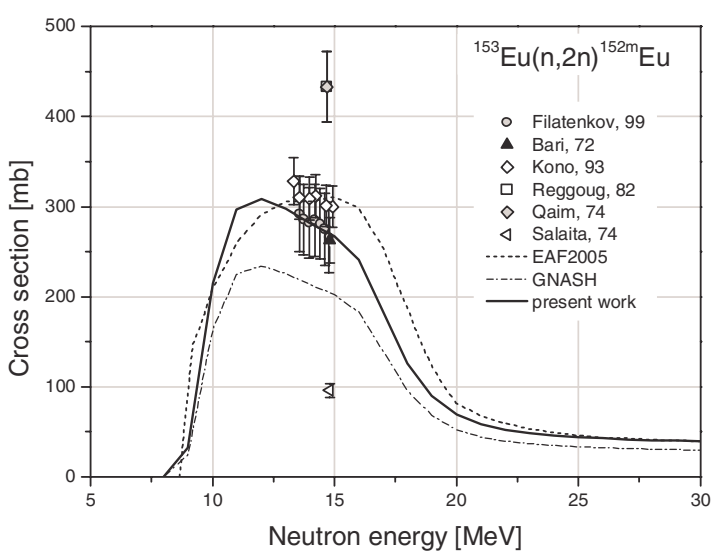

Fig. 6. ${ }^{153} \mathrm{Eu}(\mathrm{n}, 2 \mathrm{n}){ }^{152 \mathrm{~m} 1} \mathrm{Eu}$ cross section.

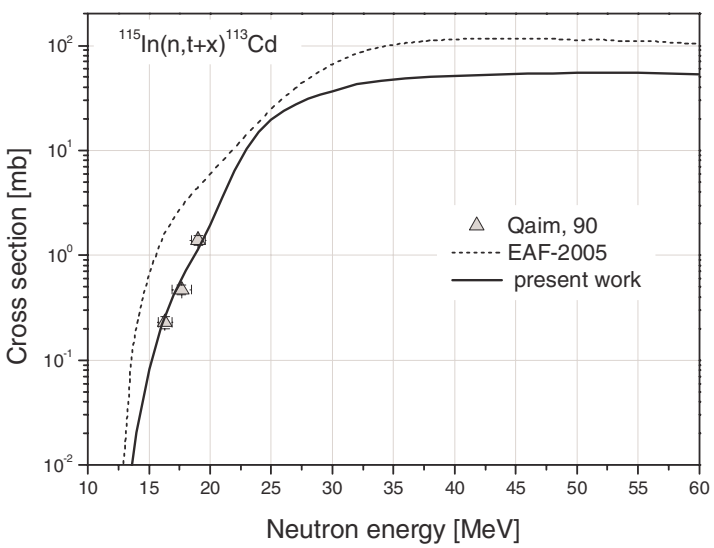

Fig. 7. ${ }^{115} \operatorname{In}(\mathrm{n}, \mathrm{x})^{113} \mathrm{Cd}$ cross section.

incident-neutron evaluations to enable further processing with standard NJOY code. To fulfill this requirement we specify only scattering radius here.

The number of the residual nuclides produced in high energy interactions rises drastically with the energy increase. The standard MT numbers of the ENDF-6 format are not sufficient to store such data. Therefore we used MT $=5$ section to store activation data in the new library. In section 


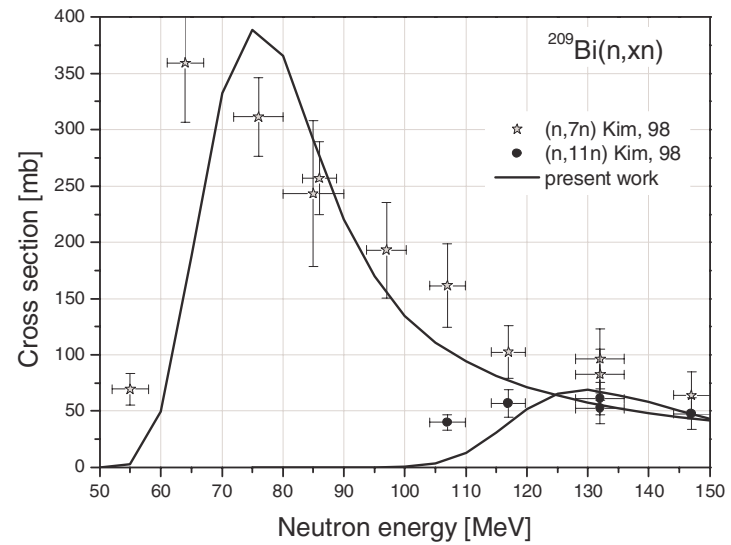

Fig. 8. ${ }^{209} \mathrm{Bi}(\mathrm{n}, 7 \mathrm{n}){ }^{203} \mathrm{Bi}$ and ${ }^{209} \mathrm{Bi}(\mathrm{n}, 11 \mathrm{n}){ }^{199} \mathrm{Bi}$ cross sections.

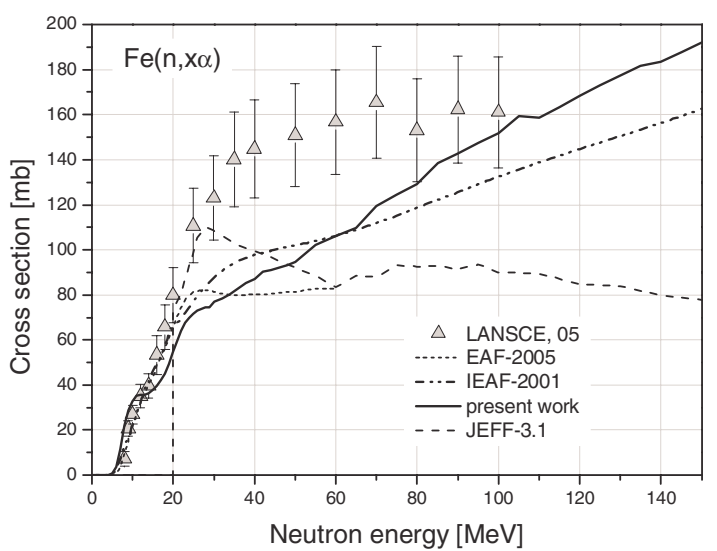

Fig. 9. He production cross section for $\mathrm{n}+\mathrm{Fe}$.

$\mathrm{MF}=3 \mathrm{MT}=5$ we present the so called lumped cross section that is a sum of all nuclide production cross sections available for a particular target nucleus. The section $\mathrm{MF}=6$ MT $=5$ contains energy dependent yields for all nuclides. An excitation function for particular isotope production can be retrieved as a product of the lumped cross section and the proper yield.

The section MF $=6 \mathrm{MT}=5$ is written using the $\mathrm{LAW}=0$ switch. For each reaction product we assign a $Z A P=$ $1000 * Z+A$ identifier followed by the LIP flag. This standard flag is used to identify the isomeric state of the residual nucleus (so LIP $=1$ is for the $1^{\text {st }}$ isomeric state). The first ZA (and the proper energy dependent yields) in $\mathrm{MF}=6$ $\mathrm{MT}=5$ is $\mathrm{ZA}=1001$ (hydrogen production) and the last one corresponds to $(\mathrm{n}, \gamma)$ reaction.

\section{References}

1. Yu.A. Korovin, A. Konobeyev, P. Pereslavtsev, A. Stankovsky, U. Fischer, U. von Mölendorf, J. Nucl. Sci. Technol. Suppl. 2 (Aug. 2002), p. 68.

2. P.G. Young, E.D. Arthur, M.B. Chadwick, Comprehensive Nuclear Model Calculations: Theory and Use of the GNASH code, Workshop on Nuclear Reaction Data and Nuclear Reactors, Trieste, Italy, April 15-May 17, 1996, p. 227.

3. T. Belgya, O. Bersillon, R. Capote, T. Fukahori, G. Zhigang, S. Goriely, M. Herman, A.V. Ignatyuk, S. Kailas, A. Koning, P. Oblozhinsky, V. Plujko, P. Young. Handbook for calculations of nuclear reaction data, RIPL-2. IAEA-TECDOC1506 (IAEA, Vienna, 2006). Available online at http://wwwnds.iaea.org/RIPL-2/.

4. J. Raynal, Notes on ECIS94, Report CEA-N-2772, Centre d'Études de Saclay CEA, Saclay, 1994.

5. R. Forest, J. Kopecky, J.-Ch. Sublet, The European Activation File: EAF-2005 cross section library, UKAEA Report, UKAEA FUS 515, 2005.

6. A. Koning, J. Delaroche, Local and global nucleon optical models from $1 \mathrm{keV}$ to $200 \mathrm{MeV}$, Nucl. Phys. A 713, 231 (2003).

7. H. An, C. Cai, Global deuteron optical model potential for the energy range up to $183 \mathrm{MeV}$, Phys. Rev. C 73, 054605 (2006).

8. P. Pereslavtsev, U. Fischer, Development of a High Energy Neutron Transmutation and Activation Data Library, EFFDOC974, NEA Data Bank, Paris, May 2006, available from http://www.nea.fr/html/dbdata/ projects/effdoc.html.

9. V. Avrigeanu, P.E. Hodgson, Global optical potentials for emitted alpha particles, Phys. Rev. C 49, 2136 (1994).

10. C. Kalbach, Phys. Rev. C 62, 044608 (2000).

11. F.D. Becchetti, G.W. Greenlees, Polarization phenomena in nuclear reactions, edited by H.H. Barshall, W. Haberly (The University of Wisconsin Press, 1971), p. 682.

12. A.J. Koning, S. Hilaire, M. Duijvestijn, Report 21297/ 04.6274/PFAI/AK/AK, Nuclear Research and Consultancy Group NRG, Pette, The Netherlands, 2004.

13. P. Pereslavtsev, U. Fischer, Nucl. Instrum. Meth. B 248, 225 (2006).

14. A. Ingemarson et al., Nucl. Phys. A 696, 3 (2001).

15. C. Kalbach, PRECO-D2: Program or calculating preequilibrium and direct reaction double differential cross sections, Report LA-10248-MS, Los Alamos National Laboratory, USA, 1985.

16. M.B. Chadwick, P.G. Young, D.C. George, Y. Watanabe, Multiple pre-equilibrium emission in Feshbach-Kerman-Koonin analyses, Phys. Rev. C 50, 996 (1994).

17. A.V. Ignatyuk, G.N. Smirenkin, A.S. Tishin, Sov. J. Nucl. Phys. 21, 255 (1975).

18. J. Kopecky, M. Uhl, Test of gamma-ray strength functions in nuclear reaction model calculations, Phys. Rev. C 41, 1941 (1990). 